\title{
El papel actual del tratamiento tradicional de la esterilidad frente a las nuevas técnicas de reproducción asistida
}

\author{
Carlos Morán $^{12}$; Raquel Huerta ${ }^{1}$; José Ignacio Madero ${ }^{4}$
}

\section{RESUMEN}

La prevalencia de los trastornos de la fertilidad está creciendo en años recientes, pero los servicios médicos para la atención de estos conocer la capacidad de resolución terapéutica de cada servicio y para seleccionar las parejas que pueden tratarse en un nivel primario de atención, o para considerar su remisión a centros de nivel ni con tecnología reproductiva. En la práctica estos trastornos se están tratando procedimientos terapéuticos para la fertilidad.

PALABRAS CLAVES: Infertilidad, niveles de atención en infertilidad, estudio de la pareja infértil.

\section{SUMMARY}

The prevalence of infertility is increasing in recent years, but the medical services to treat these problems are not available for most of the infertile couples. The prognosis for fertility is important to know the therapeutic ability of each service, and to select the couples that medical levels and the assisted reproductive technology is available only for a small popuIation sector. In general, the managed-care plans do not pay directly the infertility treatments, but they are covering in a veiled fashion some therapeutic procedures for fertility.

KEY WORDS: Infertility, levels of atention in infertility, study of the infertil couple.

\section{Introducción}

En esta revisión se abordan tres aspectos que inciden sobre el tratamiento de los trastornos de la fertilidad: 1) el aumento de la prevalencia de los problemas de fertilidad, 2) el pronóstico para la fertilidad, como mecanismo para seleccionar la atención médica, y 3) como influye la economía en el acceso al tratamiento. La hipótesis que se pretende sostener en este trabajo es que el campo de los trastornos de la fertilidad es extenso y es necesario un abordaje del problema en distintos niveles de atención médica (Tabla 1).

\section{Aumento de la prevalencia de los trastornos de la fertilidad}

En los Estados Unidos de América (en 1988), se estimó $8.4 \%$ de las mujeres de 15 a 44 años, equivalente a 4.9

1. Division of Reproductive Biology and Endocrinology, Department of Obstetrics and Gynecology, The University Of Alabama at Birmingham, Birmingham, Alabama, U. S. A.

2. Unidad de Investigación en Medicina Reproductiva, Instituto Mexicano del Seguro Social, México, D.F., México.

3. Universidad de Guanajuato, León, Guanajato, México.

4. Medifertil Programa de Medicina Reproductiva, Santafé de Bogotá, Colombia. millones de mujeres, presentaban incapacidad para conseguir embarazo. De estas mujeres, 2.7 millones presentaban esterilidad secundaria y 2.2 millones esterilidad primaria. Sin embargo, solo 1.3 millones consultaron algún servicio médico (1) También se ha calculado que $10.7 \%$ de las parejas en edad reproductiva buscan atención por trastornos de la fertilidad (2) Se piensa que desde esa fecha este problema ha crecido por diferentes razones, entre otras porque las mujeres de este grupo de edad han aumentado en mayor proporción en la población, y por tanto un mayor número de ellas busca atención médica. Además, la publicidad y difusión de las nuevas técnicas de reproducción ha atraído la atención de más parejas con el problema.

Con respecto a estos datos de frecuencias, vale la pena resaltar que dos terceras partes de las personas afectadas no buscaron atención médica, lo cual tratándose de un país desarrollado es una proporción muy grande. Se puede pensar en diferentes motivos, entre los cuales se tiene que considerar la inaccesibilidad de los servicios y el costo de ellos.

En México no se conoce la prevalencia de los trastornos de la fertilidad, ni el número de parejas que buscan atención médica por esta causa, pero si se calculan las cifras de forma aproximada para su población, extrapolándolas a los datos previos y asumiendo que una proporción 
igual de parejas tienen este problema, se encontraría que alrededor de 1.6 millones de mujeres presentan algún trastorno de la fertilidad, y que 500 mil mujeres pudieran solicitar ayuda médica anualmente para solucionar su problema de fertilidad. Si a esto se agrega que los recursos de atención médica son inferiores a los de un país desarrollado, el problema se magnifica.

En Colombia según el Censo de Población de 1993 reportado por el Departamento Administrativo Nacional de Estadística (DANE), la población total con edades entre los 15 a 44 anos es de 16'084.445, aunque no hay reportes de la Incidencia a nivel Nacional asumiendo que la incidencia es del $10 \%$ de Infertilidad, tendríamos un aproximado de 1 '608.444 personas con posibilidades de presentar problemas reproductivos quienes serían los que podrían consultar a los servicios de Infertilidad, aunque en promedio solo una tercera parte de estas parejas acuden a tratar de resolver este problema.

Se puede decir que solo desde el punto de vista de la prevalencia en los trastornos de fertilidad, el problema de la atención médica está lejos de solucionarse y que los escasos recursos especializados en técnicas de reproducción asistida son ínfimos en comparación a la gran demanda de consulta por esta causa.

\section{Pronóstico de los trastornos de fertilidad}

La pregunta más importante que surge para las parejas con un trastorno de la fertilidad es qué posibilidad tienen de lograr el embarazo. En general, no obstante la importancia que este interrogante reviste, el médico se ha acostumbrado a resolverlo de forma poco fundamentada, cuando mucho basándose en estadísticas obtenidas de la literatura médica internacional. Sin embargo, son pocos los servicios que se han preocupado de resolver seriamente esta pregunta.

En un trabajó de una cohorte de 123 parejas que consultaron por transtorno de la fertilidad (3) analizadas por tablas de vida durante un período de 30 meses, en un servicio donde no se hacían técnicas de reproducción asistida, se estudió en que forma podía influir la edad de la mujer, los eventos gestacionales previos, el tiempo de evolución del trastorno de la fertilidad y el número de factores causales de esterilidad. La tasa acumulada de embarazo (TAE) global fue del $60 \%$ a 30 meses. Las mujeres de 32 años o menores, tuvieron una TAE de $65 \%$ y aquellas demás de 32 años una TAE de $31 \%$; aunque las cifras indican cierta tendencia a mayor frecuencia de embarazos con menor edad, no hubo diferencia significativa, tal vez porque el corte se hizo a una edad joven, debido a que no había un suficiente número de mujeres arriba de los 35 años: Las mujeres con esterilidad secundaria presentaron una TAE de $88 \%$ y quienes tenían esterilidad primaria mostraron una TAE de $45 \%$, siendo marginal la diferencia estadística. Las parejas con tiempo de evolución de esterilidad de 36 meses o menos tuvieron una TAE dé $75 \%$, mientras que en las parejas con evolución mayor a 36 meses la TAE fue significativamente menor, de $43 \%$. Por último, las parejas con un solo factor causal de esterilidad presentaron una TAE de $75 \%$, con diferencia significativa en comparación a las parejas con varios factores alterados, donde la TAE fue de 47\%. Al mezclar todas estas variables en un solo valor, aplicando un sistema de puntaje, se encontró que las parejas con un mayor número de puntos tenían menor posibilidad de embarazo (Tabla 11). Un hallazgo adicional de este estudio fue que la mayor parte de embarazos se lograron en los primeros 18 meses, siendo muy escasos los embarazos en el resto del seguimiento. El factor ovárico endocrino fue donde se logró mejores resultados, con TAE de $80 \%$ a los 18 meses.

En el estudio que se menciona solo $2 \%$ de las parejas consiguieron embarazo sin ayuda de tratamiento, pero hay informes donde hasta $35 \%$ de las parejas con trastornos de la fertilidad consiguen el embarazo sin tratamiento, durante un período de seguimiento de dos a siete años (4). Estudios similares sobre pronóstico para la fertilidad se han realizado en centros donde se dispone de técnicas de reproducción asistida, encontrando que algunas de estas variables como la edad y la presencia de uno o varios factores afectan el pronóstico para el embarazo en estos grupos de pacientes (5).

Como conclusión de este estudio se puede decir que al hacer la evaluación inicial de la pareja con trastorno de la fertilidad, se puede tener una idea clara de que se puede lograr en cada caso, lo cual permite seleccionar para tratamiento a las parejas con mejor pronóstico para la fertilidad y remitir a otros niveles de atención médica con mayores recursos (entre ellos los de reproducción asistida), a las parejas con menor pronóstico. Todo servicio de fertilidad debería sistematizar sus resultados para determinar con sus propios recursos y en su población, cuales son los probables logros de su intervención.

\section{La economía en el tratamiento de los trastornos de la fertilidad}

Un aspecto de mucha importancia actualmente es como afecta el aspecto económico el tratamiento de los trastornos de la fertilidad, debido a que por su alto costo es más accesible para las clases socioeconómicas más favorecidas lo cual no resulta ético. También se considera relevante ahora el análisis inicial de costos de los procedimientos diagnósticos y terapéuticos, en cualquier sistema de atención. El otro aspecto que se discute hoy es si las aseguradoras de gastos médicos debieran cubrir los costos de un tratamiento que resulta oneroso para la mayor parte de las parejas.

\section{Análisis de costos y efectividad}

El análisis de costos involucra tanto costo y beneficio como costo y efectividad. La determinación del costo y beneficio analiza los costos de un procedimiento específico o de la aplicación de una tecnología a sus probables beneficios en términos monetarios. En contraste, la evaluación de costo y efectividad se refiere a los costos de un procedimiento y sus beneficios estimados en efectos relacionados con la salud, como vidas salvadas, disminución de la morbilidad, incapacidad evitada, etc. La tecnología médica aplicada a los trastornos de la fertilidad es costosa y desafortunadamente se aplica sin un serio análisis de costos. 
Tabla 1

ASPECTOS A CONSIDERAR SOBRE EL TRATAMIENTO DE LOS TRASTORNOS DE LA FERTILIDAD POR NIVELES DE ATENCION

Prevalencia en aumento

Incremento de la población susceptible

Más parejas consultan por este motivo

Mayor conocimiento médico

Pronóstico para el embarazo

Existen factores que inciden sobre el pronóstico

La mayor parte no requieren solución especializada

Embarazos independientes al tratamiento

Factores causales tratables por niveles de atención

El problema económico

Evaluación de costos, beneficio y efectividad

Recursos médicos disponibles

Seguridad social

Servicios privados

\section{Tabla 2}

PUNTAJE PARA DETERMINAR EL PRONOSTICO DE EMBARAZO EN PAREJAS CON TRASTORNOS DE LA FERTILIDAD

\begin{tabular}{|c|c|}
\hline Criterios & Grados \\
\hline Edad de la mujer & \\
$\leq 32$ años & 0 \\
> 32 años & 1 \\
Tipo de esterilidad & 0 \\
Secundaria & 1 \\
Primaria & 1 \\
Tiempo de evolución del trastorno & 2 \\
$\leq 36$ meses & 1 \\
$>36$ meses & 2 \\
Factores alterados & \\
Unico & \\
Múltiple & \\
\hline
\end{tabular}

Con base en este puntaje, las parejas con un total de 2 puntos tendrían aproximadamente $67 \%$ de probabilidad de embarazo, con 3 puntos $42 \%$, con 4 puntos $36 \%$, con 5 puntos $17 \%$ y con 6 puntos $0 \%$ (Morán et al. Arch Androl 36: 197, 1996).

Tabla 3

\section{CRITERIOS QUE JUSTIFICAN CONSIDERAR A LOS} TRASTORNOS DE LA FERTILIDAD DENTRO DE PLANES DE MEDICINA ASEGURADA

Definición de un punto inicial y final del trastorno Los tratamientos tienen resultados probables Es posible hacer tratamientos en períodos limitados Existen factores adicionales que afectan el resultado El costo de algunos procedimientos es elevado

Modificado de Bates GW \& Bates SR. Am J Obstet Gynecol 174: 1200,1996
Las causas demostradas más frecuentes de esterilidad son la anovulación, la alteración del factor masculino, la obstrucción o el daño de las salpinges y los problemas tuboperitoneales como la endometriosis y la enfermedad inflamatoria pélvica (6). Otras causas discutidas de los trastornos de la fertilidad son la alteración del moco cervical, la fase lútea deficiente y la esterilidad inexplicable. Muchas veces coexisten varios factores causales de esterilidad. Aunque para el estudio de estos problemas existe un número muy grande de procedimientos, en la actualidad se trata de elaborar esquemas de evaluación básica con sentido de costos y efectividad. Esta evaluación inicial justificada incluiría la historia y el examen físico de la pareja, los análisis de semen (dos o tres), la determinación de la ovulación, por curva de temperatura basal y medición de la progesterona en la fase lútea media; la histerosalpingografía, cuando existen ciclos regulares y ovulatorios, cuando hay historia sugestiva de enfermedad pélvica y cuando no hay concepción en tres ciclos ovulatorios; y la laparoscopia cuando la histerosalpingografía es negativa o sospechosa, o cuando no hay concepción en seis ciclos con inducción de la ovulación (7).

Otra alternativa que se estudia actualmente es si las técnicas de reproducción asistida pueden utilizarse como primera línea de tratamiento, en lugar del manejo tradicional descartando factores causales de esterilidad. Hasta ahora es claro que el tratamiento general corrigiendo los factores alterados, es más efectivo y tiene menor costo que las técnicas de reproducción asistida, como primer abordaje terapéutico (8).

\section{Planes de seguros médicos}

Actualmente hay una tendencia creciente a lograr que las compañías aseguradoras cubran los costos del tratamiento para los trastornos de la fertilidad. Se menciona que esto trastornos tienen los criterios para ser considerados por estos sistemas de medicina prepagada. Los criterios que se han considerado son: 1) en estos trastornos se puede establecer fácilmente un punto inicial, cuando la pareja completa un año sin concebir, y un punto final, que puede ser una edad definida de la mujer, como por ejemplo los 42 o 45 años; 2) los tratamientos tienen resultados predecibles que permiten el desarrollo de tablas estadísticas; 3) es posible desarrollar tratamientos en un período limitado; 4) hay factores que afectan el resultado como la edad y la masa corporal de la mujer; y 5) el costo de algunos procedimientos es elevado (9) (Tabla III).

En algunas partes de los Estados Unidos de América (como Maryland y Massachusetts), se ha legislado sobre la cobertura de los seguros para los trastornos de la fertilidad, pero en otros estados ha fallado la iniciativa. Por otra parte, algunas aseguradoras solo reconocen parcialmente los tratamientos, por ejemplo los que se refieren a inducción de la ovulación pero solo muy pocos cubren técnicas de reproducción asistida. Sin embargo, se sabe que muchos de los procedimientos realizados con fines de fertilidad son cobrados en forma velada a los sistemas de medicina prepagada, onsiderando el diagnóstico de la enfermedad, pero sin mención del problema de fertilidad.

Otro aspecto que se debe tener en cuenta es quien maneja los trastornos de la fertilidad. En una encuesta 
realizada en los Estados Unidos de América, 71\% de los ginecoobstetras trataban estos problemas. De ellos $82 \%$ manejaban problemas de anovulación y $23 \%$ usaban gonadotropinas. Además, $50 \%$ hacían algún tipo de cirugía para esterilidad y solo $25 \%$ de los casos eran referidos a otros servicios (10) Estos datos revelan que la gran mayoría de los trastornos de la fertilidad se resuelven en los primeros niveles de atención médica y no en los servicios más especializados.

En México se dispone de los recursos de seguridad social, los cuales no cuentan con técnicas de reproducción asistida, y solo tienen una cobertura limitada a una parte de la población. Por otra parte, existen los servicios privados, los cuales van desde el simple consultorio del médico especialista hasta los servicios más complejos de reproducción asistida, solo disponibles para un minúsculo sector de la población. La cobertura de las compañías de seguros médicos a los trastornos de la fertilidad incrementaría la accesibilidad de los servicios más especializados a un sector mayor de la población.

En el caso de Colombia, la Ley 100 de 1993 no contempla dentro de su cobertura los tratamientos de reproducción humana. Actualmente existen centros de fertilidad privados que brindan este tipo de tratamientos con muy buenos resultados.

\section{Conclusiones}

1. La prevalencia de los trastornos de la fertilidad está en aumento, y los servicios médicos para la atención de estos problemas no se encuentran al alcance de la mayor parte de las parejas que los necesitan.

2. El pronóstico de los trastornos de la fertilidad es importante como método para conocer la capacidad de resolución terapéutica de cada servicio y como mecanismo de selección de las parejas susceptibles de tratamiento en un nivel de atención, o en caso contrario, para considerar su remisión a niveles superiores de tecnología reproductiva.

3. En la práctica, estos trastornos se están tratando en los primeros niveles de atención médica y las técnicas de reproducción asistida solo son accesibles para un pequeño sector de la población.

4. Las compañías de seguros médicos no reconocen directamente los tratamientos para estos problemas, pero cubren veladamente algunos procedimientos. Convendría que los sistemas de medicina prepagada consideraran a los tratamientos de los trastornos de la fertilidad y se legislara sobre estos aspectos.

\section{REFERENCIAS}

1. Mosher WD, Pratt WF. Fecundity and infertility in the United States, 1965-1988. Advance data from vital and health statistics No. 192. Hyattsville, Md. PHS 1990; 91: 1250.

2. Wilcox LS, Mosher WD. Use of infertility services in the United States. Obstet Gynecol 1993; 82: 122-127.

3. Morán C, García-Hernández E, Carranza-Lira S, Cortés A, Varón J, Merino G, Bermúdez JA. Prognosis for fertility analyzing different variables in men and women. Arch Androl 1996; 36: 197-204.

4. Collins JA, Wrixon W, Janes LB, Wilson EH. Treatmentindependent pregnancy among infertile couples. N Engl J Med 1983; 309: 1201-1206.

5. Check JH, Lurie D, Callan C, Baker A, Benfer K. Comparison of the cumulative probability of pregnancy after in vitro fertilizationembryo transfer by infertility factor and age. Fertil SteriI1994; 61: 257-261.
6. Jones HW, Toner JP. The infertile couple. N Engl J Med 1993; 23: 1710-1715.

7. Knochenhauer ES, Azziz R. Evaluation of infertility: a cost effective approach. Ref Gynecol Obstet 1997; 5: 315-320.

8. Karande YC, Kom A, Morris R, Rao R, Balin M, Rinehart J, Dohn $\mathrm{K}$, Gleicher N. Prospective randomized trial comparing the outcome and cost of in vitro fertilization with that of a traditional treatment algorithm as first-line therapy for couples with infertility. Fertil Steril 1999; 71: 468-475.

9. Bates GW, Bates SR. The economics of infertility: developing an infertility managed-care plan. Am J Obstet Gynecol 1996; 174: 12001207.

10. Wentz AC. News of the Society of Reproductive Endocrinologists. Fertility News 1992; 26: 4. 